\title{
Efficacy of alogliptin combined with motor imagery under hyperbaric oxygen in diabetic nephropathy with silent cerebral infarction
}

\author{
DANYAN CHEN ${ }^{1}$, XIAOLONG HUANG $^{2}, \mathrm{HUA} \mathrm{GAN}^{3}, \mathrm{XIAOGANG} \mathrm{DU}^{3}$, \\ SONG LU ${ }^{1}$, RONGXI HUANG ${ }^{1}$, KE LIU $^{1}$ and BINGHAN ZHANG ${ }^{1}$ \\ ${ }^{1}$ Department of Endocrinology and Nephrology, The Chongqing General Hospital, Chongqing 400013; \\ ${ }^{2}$ Department of Neurosurgery, No. 324 Hospital of PLA, Chongqing 400028; ${ }^{3}$ Department of Nephrology, \\ The First Affiliated Hospital of Chongqing Medical University, Chongqing 400016, P.R. China
}

Received July 31, 2017; Accepted September 14, 2017

DOI: $10.3892 /$ br.2017.983

\begin{abstract}
In the present study, we evaluated the curative effect of dipeptidyl peptidase-IV (DPP-IV) inhibitor alogliptin combined with motor imagery under hyperbaric oxygen in diabetic nephropathy (DN) with silent cerebral infarction (SCI). Two-hundred newly diagnosed DN patients with and without SCI were included. The SCI patients were divided into two treatment groups: Alogliptin (A group, $n=50$ ) and alogliptin combined with motor imagery under hyperbaric oxygen (B group, $n=50$ ). The degrees of neurocognitive dysfunction were evaluated at baseline and after 6 months of treatment. Thromboelastograms (TEGs) mapping were conducted. Serum glycoprotein VI (GPVI) mRNA expression and urine 11-DH-TXB ${ }_{2}$ levels were determined. Compared to group A patients, the severity of neurofunctional defects, GPVI mRNA expression and 11-DH-TXB ${ }_{2}$ levels were significantly lower in group $\mathrm{B}(\mathrm{P}<0.05)$, while comprehensive, MoCA scores were higher in group B. The MoCA subscores of visuospatial/executive function, attention and concentration were significantly higher compared to group $\mathrm{A}(\mathrm{P}<0.05)$. The sub-scores of computation, abstract thinking, language competence, memory and orientation were also higher in group B but the differences were not significant $(\mathrm{P}>0.05)$. TEG indexes were improved in both groups after treatment as manifested by increased $R$ and $K$ values, but there was significant improvement in group B. Intra-group comparisons revealed a time-dependent effect of treatment. In conclusion, the treatment of alogliptin combined with motor imagery under hyperbaric oxygen can better promote thrombolysis
\end{abstract}

Correspondence to: Dr Danyan Chen, Department of Endocrinology and Nephrology, The Chongqing General Hospital, 312 Zhongshan First Road, Yuzhong, Chongqing 400013, P.R. China E-mail: clioyoyo@163.com

Key words: diabetic nephropathy, silent cerebral infarction, alogliptin, hyperbaric oxygen, motor imagery absorption, restore brain damage and improve neurocognitive function in DN with silent cerebral infarction.

\section{Introduction}

Approximately 5.23-11.04\% of newly diagnosed type 2 diabetes mellitus cases are complicated by cerebrovascular disorders (1). Currently, CI is divided into symptomatic and silent cerebral infarction (SCI) according to clinical manifestations. Clinically, CI often occurs after transient ischemic attack (TIA) and shows no obvious symptoms or signs due to the small territory of infarction or involvement outside areas associated with easily observable functional manifestations. Thus, SCI is often misdiagnosed or missed in routine examination. However, SCI may be detected by brain computed tomography (CT) or magnetic resonance imaging (MRI) $(2,3)$. Recurrent SCI may cause cognitive decline, symptomatic cerebral infarction, vascular dementia or Parkinsonism, affecting patient quality of life and increasing family and social burdens (4).

The inhibition of dipeptidyl peptidase-IV (DPP-IV) and increase of the activity of endogenous glucagon-like peptide-1 are new therapeutic targets of diabetes mellitus. DPP-IV inhibitors have biological effects for reducing the incidence of ischemic cerebral infarction and improving the cognitive function, in addition to a hypoglycemic effect $(5,6)$. However, there is no study on the role of DPP-IV inhibitor alogliptin in the treatment of diabetes nephropathy (DN) complicated with silent cerebral infarction. Hyperbaric oxygen is one of the effective methods to treat cerebral infarction (7). However, due to the special therapeutic environment, patient compliance is poor and the curative effect is easily affected. It was reported that stroke patients who received motor imagery training reached a certain degree of athletic and cognitive rehabilitation (8).

Thus, the present study is focused on whether alogliptin in combination with motor imagery in a hyperbaric oxygen chamber can effectively reduce blood glucose, overcome the aforementioned shortcomings and improve the function impairment of DN patients complicated with SCI. 


\section{Materials and methods}

Patients. We enrolled 200 patients who where newly diagnosed with diabetic nephropathy (DN) from the Chongqing General Hospital and the First Affiliated Hospital of Chongqing Medical University.

DN was diagnosed clinically if one or more of the following criteria were fulfilled: i) Histological diagnosis by renal biopsy; ii) presence of diabetic retinopathy; and iii) history of type 2 diabetes mellitus at least 3 years before enrollment. All participants had a glomerular filtration rate (eGFR) of $>30 \mathrm{ml} / \mathrm{min} / 1.73 \mathrm{~m}^{2}$. The patients were divided into the SCI group and without SCI (NSCI) group according to radiological data and clinical features. The SCI group patients were divided into two treatment groups: Alogliptin (group A, n=50) and alogliptin combined with motor imagery under hyperbaric oxygen (group B, n=50). Diagnostic criteria of SCI $(9,10)$ were: i) No neurological symptom or sign; ii) low signals on MRI T1WI and high signals on T2WI, lesion diameter of $>3 \mathrm{~mm}$ and no high signal on MRI DWI. Inclusion criteria for the SCI group were: i) Daily life and social activities were not obviously affected; ii) SCI lesion was confirmed by MRI and no encephalatrophy was noted; iii) the patients were right-handed.

Exclusion criteria were: i) Patients with a definite history of cerebral infarction and dementia; ii) patients with a definite history of other central nervous system diseases such as infection and demyelinating disease; iii) patients with severe physical diseases; iv) patients with a definite history of mental and psychological diseases such as schizophrenia and major depression; v) alcohol or drug addicts; vi) patients unable to complete the scale of cognitive function due to severe diseases or physical disabilities; vii) patients with allergic constitution; viii) patients with active bleeding or bleeding tendency; ix) patients on non-steroid anti-inflammatory drugs, glucocorticoids, thyroid hormone tablets and other drugs; and x) patients with liver disease. NSCI patients were those without cerebral infarction or patients with symptomatic cerebral infarction. After the screening period, patients received DPP-IV inhibitor alogliptin (25 mg once daily; Takeda Pharmaceutical Company Ltd., Japan), in addition to continuing their anticoagulation background treatment. The patients in group B underwent motor imagery in the hyperbaric oxygen chamber after taking alogliptin. Informed consent was obtained from all the patients. The protocol was registered with the Chinese Clinical Trial Registry (ChiCTR-INR-17012590). The hospital Ethics Committee had reviewed our study.

Treatment of motor imagery in the hyperbaric oxygen chamber. A hyperbaric oxygen chamber (Haux-Life-Support $\mathrm{GmbH}$, Baden-Wurttemberg, Germany) for 34 patients was used. At the pressure of $0.2 \mathrm{MPa}$, the patients inhaled pure oxygen using a mask for $30 \mathrm{~min}$ and rested for $10 \mathrm{~min}$ between two episodes of oxygen inhalation. The pressure was increased and pressured at a uniform speed for $15 \mathrm{~min}$. The hyperbaric oxygen therapy was performed once a day and 10 days were a course of treatment. There was an interval of 5 days between each course of treatment. The patients were required to perform intermittent motor imagery $(11,12)$ during the hyperbaric oxygen therapy. After the motor imagery, the patients were asked to open their eyes, move their extremities and joints following the rhythms of music and carry out alternative muscular tension-relaxation activities for 5-10 min.

Neurocognitive dysfunction degree and clinical curative effect evaluation. Two physicians carried out the evaluation according to the National Institutes of Health Stroke Scale (NIHSS) (13). Scores of 0-1 were considered normal or near-normal range and 1-4 were considered mild. Scores of 5-15 were considered moderate; $15-20$ were considered moderate-severe; and 21-42 were severe. Evaluation criteria of the clinical curative effect were: Basic cure (decrease of scores $>90 \%$ ), significant change (decrease of scores 46-89\%), change (decrease of scores $18-45 \%$ ), no change (decrease of scores $\leq 17 \%$ ) and deterioration (scores increase). When the scores were reduced by $>18 \%$, it indicated that the treatment was effective. The total effective rate was calculated as: (cases of basic cure + cases of significant change + cases of change)/total cases $\times 100 \%$.

Montreal cognitive assessment (MoCA) (14) was performed from eight aspects of visuospatial/executive ability, attention, memory, language, abstraction, naming, calculation and orientation. The scale was completed within $10 \mathrm{~min}$ and the total scores were 30 . Scores of $>26$ were considered normal cognition. In case of the education years being $\leq 12$ year, the scores were added to be 1 .

Thromboelastograms (TEGs) mapping. Blood samples were collected from the antecubital vein and tested with Thrombelastograph Analyzer TEG-500 (15). The tested parameters included: i) Reaction time (R), which indicated the time from the test start to the formation of the clot or fibrin. Normal $\mathrm{R}$ values ranged between 5 and $10 \mathrm{~min}$; ii) clotting time (K), considered from the clotting start to the time when TEG amplitude reached $20 \mathrm{~mm}$. Normal $\mathrm{K}$ values ranged between 1 and $3 \mathrm{~min}$; iii) $\alpha$-angle, which was the angle between the tangent line and the horizontal line of the maximum curve when TEG reached the maximum curve from clot formation, representing the speed of clot formation and the speed of hemocoagulase formation. Normal $\alpha$-angle was between 53 and $72^{\circ}$; iv) maximum amplitude (MA), which was the maximum solidness of thrombus, reflecting the absolute clot intensity. Normal values were between 50 and $70 \mathrm{~mm}$; v) clot strength (G): $\mathrm{G}$ value was a measure of clot strength or clot firmness, and was calculated based on the amplitude value until the MA was reached. Normal G values were between 4.6 and $11 \mathrm{kDa}$; vi) coagulation index (CI): $-3<$ normal $<+3$; index $<-3$ indicated low coagulation; index $>-3$ indicated high coagulation. If two or more of the following conditions were met, it was diagnosed as hypercoagulable state: $\mathrm{R}$ and $\mathrm{K}$ were shortened and $\alpha$-angle and/or MA were increased.

Reverse transcriptase-quantitative polymerase chain reaction (RT-qRCR) detection glycoprotein VI (GPVI) mRNA expression. Total RNA was extracted with TRIzol (Takara Bio, Inc., Otsu, Japan) according to the manufacturer's instructions. For RT-qPCR, cDNA was synthesized using the PrimeScript RT-PCR kit (Takara Bio, Inc.). The primers (Shanghai Biotechnology, Shanghai, China) used to detect human GPVI and $\beta$-actin (internal control) were: GPVI forward, 5'-GCCAA GCTATTGCGACATGA-3' and reverse, 5'-AAAAGAATCT CAATGTCCGAGACTTT-3'; $\beta$-actin forward, 5'-GGAGCGA 
Table I. Basic clinical and laboratory characteristics of diabetic nephropathy with and without SCI.

\begin{tabular}{|c|c|c|c|}
\hline Characteristics & NSCI group $(n=100)$ & SCI group $(n=100)$ & P-value \\
\hline Age (years) & $61 \pm 5$ & $62 \pm 4$ & 0.793 \\
\hline Sex (male:female) & $51: 49$ & $50: 50$ & 0.886 \\
\hline Smoking & $14(14 \%)$ & $15(15 \%)$ & 0.852 \\
\hline Hyperlipidemia & $8(8 \%)$ & $10(10 \%)$ & 0.847 \\
\hline Atrial fibrillation & $5(5 \%)$ & $7(7 \%)$ & 0.765 \\
\hline Systolic pressure (mmHg) & $131.66 \pm 4.52$ & $133.00 \pm 5.04$ & 0.694 \\
\hline Diastolic pressure $(\mathrm{mmHg})$ & $79.92 \pm 4.69$ & $81.20 \pm 3.88$ & 0.703 \\
\hline Duration of diabetes (year) & $3.70 \pm 0.80$ & $3.50 \pm 1.10$ & 0.778 \\
\hline Duration of diabetic nephropathy (year) & $0.50 \pm 0.10$ & $1.10 \pm 0.00^{\mathrm{a}}$ & 0.049 \\
\hline Intima-media thickness (mm) & $1.18 \pm 0.06$ & $1.41 \pm 0.08^{\mathrm{a}}$ & 0.040 \\
\hline HOMA-IR & $3.85 \pm 0.72$ & $5.86 \pm 0.51^{\mathrm{a}}$ & 0.045 \\
\hline НОМА- $\beta$ & $36.50 \pm 2.42$ & $28.60 \pm 5.36^{\mathrm{a}}$ & 0.043 \\
\hline Glycosylated hemoglobin A1c (\%) & $6.81 \pm 0.25$ & $7.09 \pm 0.05$ & 0.848 \\
\hline Fibrinogen & $3.32 \pm 0.38$ & $4.66 \pm 0.35^{\mathrm{a}}$ & 0.048 \\
\hline $\mathrm{ABI}$ & $0.91 \pm 0.06$ & $0.55 \pm 0.07^{\mathrm{a}}$ & 0.049 \\
\hline Body fat mass $(\%)$ & $26.10 \pm 4.09$ & $31.80 \pm 4.10^{\mathrm{a}}$ & 0.032 \\
\hline
\end{tabular}

Values are given as mean \pm standard deviation or number. ${ }^{\mathrm{a}} \mathrm{P}<0.05$, SCI vs. NSCI. HOMA-IR $=$ fasting glucose $\mathrm{x}$ fasting insulin/22.5; HOMA- $\beta=20 \mathrm{x}$ fasting insulin/(fasting glucose - 3.5); ABI = ankle vs. systolic pressure/brachial arterial systolic pressure; body fat mass $($ male $)=1.2 \times$ BMI $+0.23 \times$ age -16.2 ; body fat mass $($ female $)=1.2 \times$ BMI $+0.23 \times$ age $-15.4 ; \mathrm{BMI}=$ weight $/$ height $^{2}$. SCI, silent cerebral infarction; HOMA-IR, homeostasis model assessment insulin resistance; HOMA- $\beta$, homeostasis model assessment for $\beta$-cell function; $\mathrm{ABI}$, ankle brachial index.

GATCCCTCCAAAAT-3' and reverse, 5'-GGCTGTTGTCAT ACTTCTCATGG-3'. The cDNAs were amplified using the following thermal cycle: Denaturation at $95^{\circ} \mathrm{C}$ for $1 \mathrm{~min}$, followed by 35 cycles of denaturation at $95^{\circ} \mathrm{C}$ for $30 \mathrm{sec}$, annealing for $1 \mathrm{~min}$ at $58^{\circ} \mathrm{C}$, polymerization for $1 \mathrm{~min}$ at $70^{\circ} \mathrm{C}$, and brief detection at $70^{\circ} \mathrm{C}$. The signal was expressed relative to $\beta$-actin (relative ratio $=$ average copy number of target gene in the sample/average copy number of $\beta$-actin).

Enzyme-linked immunosorbent assay (ELISA) detection levels of $11-\mathrm{DH}-\mathrm{TXB}_{2}$. The production of 11-DH-TXB $\mathrm{T}_{2}$ in the urine after $24 \mathrm{~h}$ (overnight) of treatment was quantified with ELISA detection kits (Quantikine; R\&D Systems, Abingdon, UK) according to the manufacturer's instructions. Optical densities were measured at $450 \mathrm{~nm}$. Each assay was performed in triplicate.

Statistical analysis. Data were analyzed with SPSS v23.0 statistical software (SPSS, Inc., Chicago, IL, USA). Values are expressed as mean \pm standard deviation throughout the text and figures, and the enumeration data were expressed by Chi-square test. Comparison among groups was conducted by t-test. $\mathrm{P}<0.05$ was considered to indicate a statistically significant difference.

\section{Results}

Basic clinical characteristics of patients. Among DN patients, there was no significant difference in age, sex and risk factors (history of smoking, atrial fibrillation, hyperlipidemia, hypertension, duration of diabetes and $\mathrm{HbA1c}$ ) between the SCI and NSCI groups $(\mathrm{P}>0.05)$. However, there was significant difference in homeostasis model assessment insulin resistance (HOMA-IR), homeostasis model assessment for $\beta$ cell function (HOMA- $\beta$ ) between the SCI and NSCI groups $(\mathrm{P}<0.05)$. IMT, fibrinogen (FIB), and body fat mass of the NSCI group were lower than those of the SCI group with significant inter-group difference $(\mathrm{P}<0.05)$. Ankle brachial index $(\mathrm{ABI})$ of the NSCI group was higher than that of SCI group, also with significant inter-group difference $(\mathrm{P}<0.05)$ (Table I).

MRI features and clinical manifestation. The infarction foci of DN patients with SCI presented as different sizes and shapes. Among SCI patients, 30 patients had lacunar infarction (diameter $\leq 20 \mathrm{~mm}$ ), 22 had a single lesion and $28 \mathrm{had}$ multiple lesions. The infarction foci of 28 patients were identified in the basal ganglia region and others were evident in the internal capsule, ventricle, thalamus, left temporal lobe, radial crown, bridge brain and insular lobe. Generally, SCI patients had no focal neurological signs. Non-specific signs included headache, dizziness, fatigue, impaired memory, slow response and subjective feeling and limb numbness (Fig. 1).

Neurocognitive dysfunction degree score and clinical curative effect. Compared with the NSCI group, the neurological deficit scores of the SCI group were increased $(4.43 \pm 1.12$ vs. $8.48 \pm 3.69, \mathrm{P}<0.05)$. Before treatment, the comparison between groups $\mathrm{A}$ and $\mathrm{B}$ showed no significant difference in neurological deficit scores $(8.47 \pm 3.72$ vs. $8.49 \pm 3.68, \mathrm{P}>0.05)$. Six months after treatment, the neurological deficit scores of group $B$ were lower than those of group A, with a significant difference 
Table II. Clinical curative effect of SCI patients between the treatment groups.

\begin{tabular}{lccccccc}
\hline Groups & $\mathrm{n}$ & Basic cure & Significant change & Change & No change & Deterioration & Total effective rate $(\%)$ \\
\hline A & 50 & 4 & 18 & 8 & 18 & 2 & 60 \\
B & 50 & 9 & 26 & 6 & 9 & 0 & 82 \\
\hline
\end{tabular}

SCI, silent cerebral infarction.

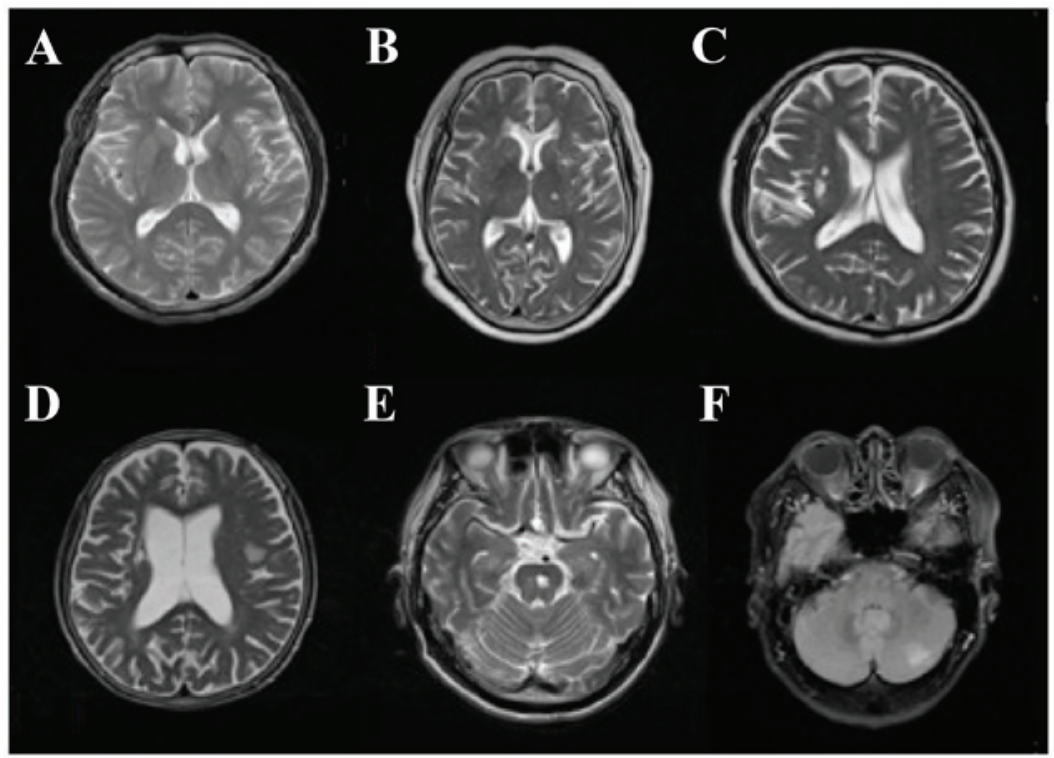

Figure 1. MRI features in diabetic nephropathy patients complicated with silent cerebral infarction. (A) Normal control, (B) multiple lacunar infarction, (C) right basal ganglia region infarction, (D) left temporal lobe and insular lobe infarction, (E) pontine infarction, and (F) left cerebellar infarction.

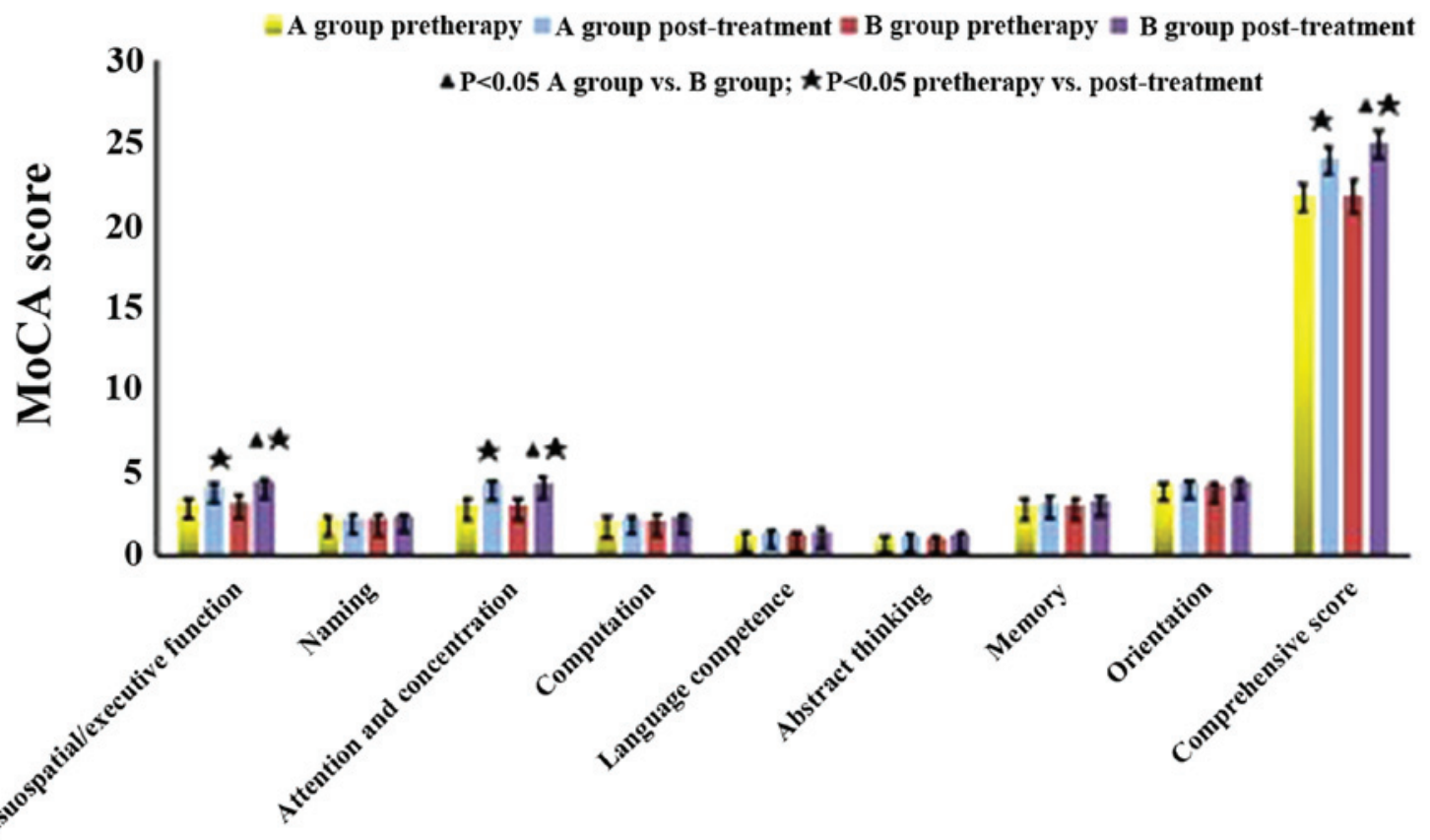

Figure 2. Comparison of Montreal cognitive assessment score between treatment groups of diabetic nephropathy with silent cerebral infarction.

(6.88 \pm 2.20 vs. $4.58 \pm 2.17, \mathrm{P}<0.05)$. The inter-group compared before and after treatment showed that the neurological deficit was improved $(\mathrm{P}<0.05)$. As shown in Table II, the curative effect of group B was better than that of group A $(\mathrm{P}<0.05)$. 
MoCA score. Compared with the NSCI group, the MoCA scores of the SCI group were decreased $(\mathrm{P}<0.05)$. As shown in Table III, before treatment, the comparison between groups $\mathrm{A}$ and $\mathrm{B}$ showed no significant difference in MoCA scores $(\mathrm{P}>0.05)$. MoCA scores of group B were higher than those of group A after a 6-month treatment. The subscores of visuospatial/executive function, attention and concentration and comprehensive score were significantly increased $(\mathrm{P}<0.05)$, while the subscores of computation, abstract thinking, language competence, memory, orientation and memory were also increased, albeit the difference was not statistically significant $(\mathrm{P}>0.05)$ (Fig. 2).

TEGs. As shown in Fig. 3, there was no statistical significance of single and multiple lesions in SCI patients $(\mathrm{P}>0.05)$. Before treatment, the decreased $\mathrm{R}$ and $\mathrm{K}$ values, the increased MA value, $\alpha$-angle and $\mathrm{G}$ value as well as the prolonged $\mathrm{CI}$ indicated DN patients with silent cerebral infarction were in an hypercoagulable state (Table IV). Compared with those before treatment, the TEG indexes of both groups after treatment were improved, manifesting as increased $\mathrm{R}$ and $\mathrm{K}$ values, decreased MA value, $\alpha$-angle and G, and shortened CI. The comparison between groups $\mathrm{A}$ and $\mathrm{B}$ after treatment showed significant difference $(\mathrm{P}<0.05)($ Table $\mathrm{V})$.

mRNA expression of GPVI and level of 11-DH-TXB ${ }_{2}$. Compared with the NSCI group, GPVI expression and $11-\mathrm{DH}-\mathrm{TXB}_{2}$ level of the SCI group were increased (GPVI: $17.50 \pm 4.50$ vs. $20.86 \pm 4.62, \mathrm{P}<0.05 ; 11-\mathrm{DH}-\mathrm{TXB}_{2}$ : $43.21 \pm 6.38$ vs. $58.39 \pm 7.22, \mathrm{P}<0.05)$. Before treatment, the comparison between groups $\mathrm{A}$ and $\mathrm{B}$ showed no significant difference in GPVI expression and 11-DH-TXB ${ }_{2}$ levels (GPVI: $20.91 \pm 4.83$ vs. $20.80 \pm 4.66, \mathrm{P}>0.05 ; 11-\mathrm{DH}-\mathrm{TXB}_{2}: 58.55 \pm 7.15$ vs. $58.28 \pm 7.34, \mathrm{P}>0.05)$. Six months after treatment, the GPVI expression and 11-DH-TXB ${ }_{2}$ level of group B were lower than those of group A, with a significant difference (GPVI: $18.11 \pm 4.26$ vs. $14.81 \pm 4.08, \mathrm{P}<0.05 ; 11-\mathrm{DH}-\mathrm{TXB}_{2}: 46.80 \pm 6.69$ vs. $39.30 \pm 6.25, \mathrm{P}<0.05)$. As shown in the inter-group comparison before and after treatment, the difference of GPVI expression and 11-DH-TXB ${ }_{2}$ level was statistically significant $(\mathrm{P}<0.05)$ (Fig. 4).

\section{Discussion}

$\mathrm{DN}$ is one of the independent risk factors for cerebral infarction. The clinical manifestations of cerebral infarction perform as SCI and recurrent transient ischemic attack. SCI is short for silent cerebral infarction and it is known as occult cerebral infarction or subclinical stroke $(16,17)$. It is a particular type of cerebral infarction and the most common SCI is lacunar infarction, which has a small range of cerebral infarction or involved cerebral tissues far from the functional area $(18,19)$. The abovementioned study results are consistent with those obtained in the present study.

The prevention and treatment of DN complicated with SCI has become a research hotspot. Incretin drugs are new hypoglycemic drugs, mainly including glucagon-like peptide 1 (GLP1) receptor agonist and DPP-IV inhibitors. DPP-IV inhibitors have other biological effects besides a hypoglycemic effect. The combination of incretin drugs and GLP-1R may activate adenylate cyclase, upregulate cyclic adenosine monophosphate 
Table IV. Comparison of TEG parameters of single lesion and multiple lesions in SCI patients.

\begin{tabular}{lcccrrr}
\hline Parameters & $\mathrm{R}(\mathrm{min})$ & $\mathrm{K}(\mathrm{min})$ & $\alpha$-angle $(\circ)$ & $\mathrm{MA}(\mathrm{mm})$ & $\mathrm{G}(\mathrm{kDa})$ & $\mathrm{CI}$ \\
\hline Single lesion & $5.55 \pm 1.53$ & $1.52 \pm 0.82$ & $68.92 \pm 11.0$ & $72.07 \pm 5.71$ & $11.60 \pm 1.74$ \\
Multiple lesions & $5.83 \pm 1.39$ & $1.57 \pm 0.68$ & $69.89 \pm 9.63$ & $73.35 \pm 5.32$ & $11.43 \pm 2.01$ & $2.20 \pm 0.90$ \\
\hline
\end{tabular}

Values are given as mean \pm standard deviation or number. TEG, thromboelastogram; SCI, silent cerebral infarction; R, reaction time; K, clotting time; MA, maximum amplitude; G, clot strength; CI, coagulation index.

Table V. Comparison of TEG parameters between treatment groups of diabetic nephropathy with SCI.

\begin{tabular}{|c|c|c|c|c|c|c|c|}
\hline Groups & Treatment & $\mathrm{R}(\min )$ & $\mathrm{K}(\min )$ & $\alpha$-angle (॰) & $\mathrm{MA}(\mathrm{mm})$ & $\mathrm{G}(\mathrm{kDa})$ & $\mathrm{CI}$ \\
\hline \multirow[t]{2}{*}{$A(n=50)$} & Pretherapy & $4.04 \pm 0.82$ & $1.20 \pm 0.19$ & $72.15 \pm 8.91$ & $75.06 \pm 5.46$ & $12.30 \pm 1.42$ & $2.93 \pm 0.52$ \\
\hline & Post-treatment & $6.20 \pm 0.84^{\mathrm{b}}$ & $1.96 \pm 0.23^{\mathrm{b}}$ & $63.22 \pm 9.04^{\mathrm{b}}$ & $70.40 \pm 5.35^{\mathrm{b}}$ & $10.12 \pm 1.38^{\mathrm{b}}$ & $1.85 \pm 0.32^{\mathrm{b}}$ \\
\hline \multirow[t]{2}{*}{$B(n=50)$} & Pretherapy & $4.05 \pm 0.86$ & $1.21 \pm 0.20$ & $72.00 \pm 9.00$ & $75.00 \pm 5.62$ & $11.93 \pm 1.67$ & $2.94 \pm 0.50$ \\
\hline & Post-treatment & $6.78 \pm 0.32^{\mathrm{a}, \mathrm{b}}$ & $2.28 \pm 0.19^{\mathrm{a}, \mathrm{b}}$ & $60.38 \pm 9.21^{\mathrm{a}, \mathrm{b}}$ & $66.90 \pm 5.93^{\mathrm{a}, \mathrm{b}}$ & $8.62 \pm 1.62^{\mathrm{a}, \mathrm{b}}$ & $1.56 \pm 0.39^{\mathrm{a}, \mathrm{b}}$ \\
\hline
\end{tabular}

Values are given as mean \pm standard deviation or number. ${ }^{\mathrm{a}} \mathrm{P}<0.05$, group $\mathrm{A}$ vs. group $\mathrm{B}$; ${ }^{\mathrm{b}} \mathrm{P}<0.05$, pretherapy vs. post-treatment. TEG, thromboelastogram; SCI, silent cerebral infarction; R, reaction time; K, clotting time; MA, maximum amplitude; G, clot strength; CI, coagulation index .

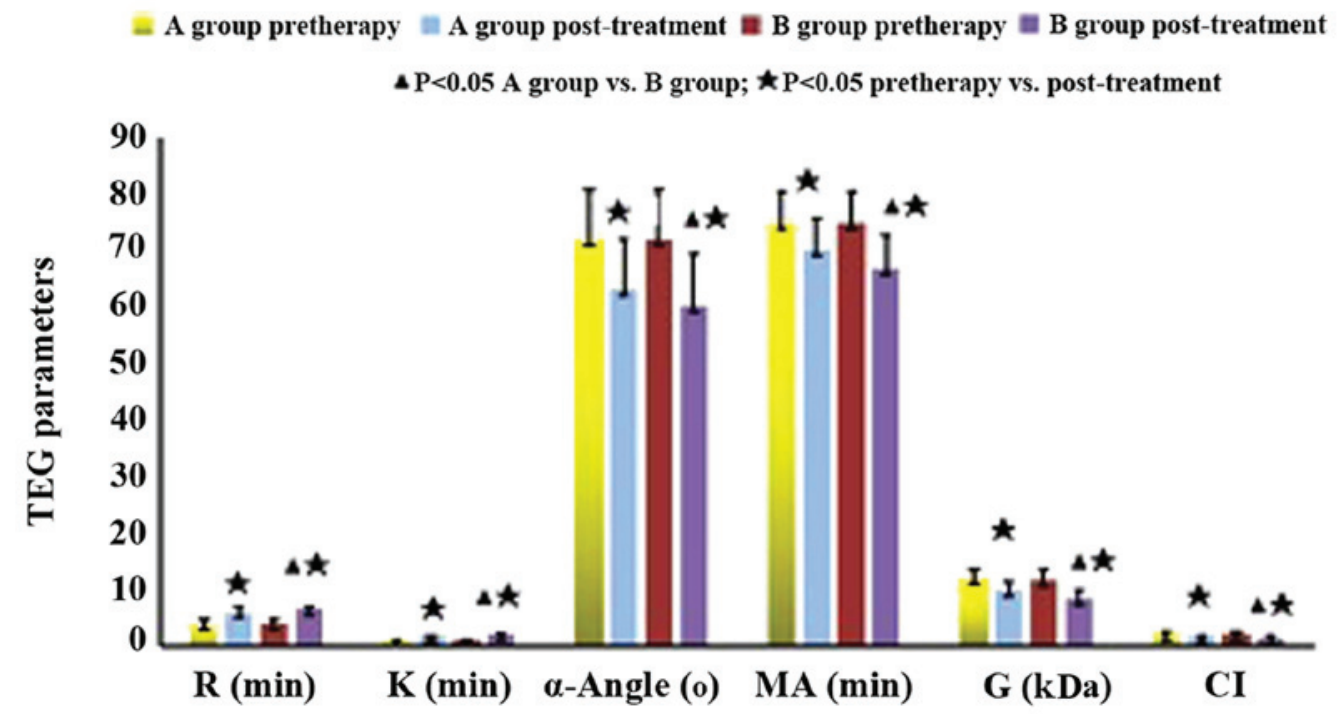

Figure 3. Comparison of TEG parameters between treatment groups of diabetic nephropathy with silent cerebral infarction. TEG, thromboelastogram.

levels, trigger $\mathrm{Ca}^{2+}$ inflow, enhance synaptic plasticity, increase neurotransmitter release and improve learning, memory and cognitive function $(20,21)$. Hyperbaric oxygen therapy may increase the oxygen saturation of patients, reduce intracranial pressure, relieve inflammatory reaction and edema degree, decrease infarction size (22), use 'reverse steal phenomenon' to increase blood and oxygen supply in the focal area, increase the mitochondrial membrane permeability of brain cells, reduce neutrophil apoptosis and promote neural functional recovery and regeneration (23). Thus, it is an effective means to treat cerebral infarction. However, patients may generate fear, anxiety irritability and other negative emotions during the treatment due to the closed environment. Therefore, we utilized the DPP-IV inhibitor alogliptin in combination with motor imagery in a hyperbaric oxygen chamber to treat DN patients with SCI. Motor imagery may be used to reinforce and improve exercise plans, enhance sensory information input, promote active latent pathways and dormant synapse, accelerate reperfusion of ischemic penumbra and improve cerebral blood flow by repeatedly simulating and rehearsing exercises in heart without obvious body movement according to the exercise plans stored in a certain active brain area in order to repair the neurologic impairment to a certain degree $(24,25)$.

The Montreal cognitive assessment (MoCA) is more sensitive in testing mild vascular cognitive impairment than MMSE (26-28). In the present study, the neurocognitive dysfunction of SCI patients was more severe than that of NSCI patients. The naming and language functioning capabilities of 

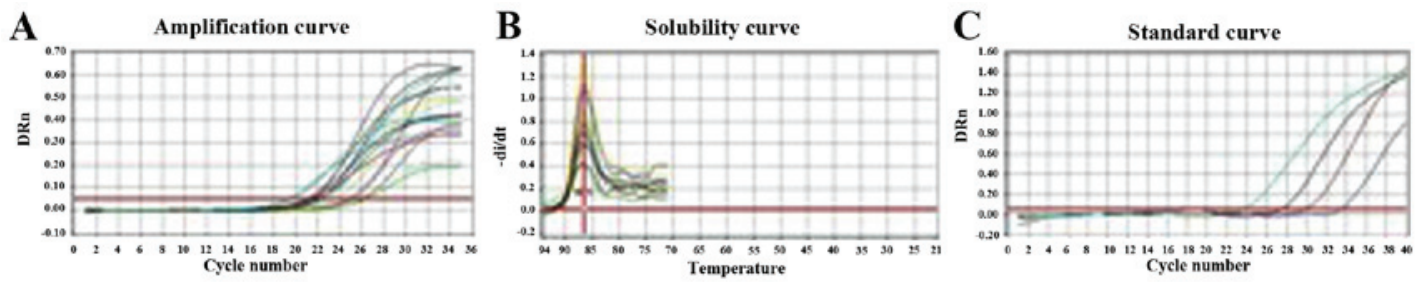

$₫ \mathrm{P}<0.05$ SCI group vs. NSCI group
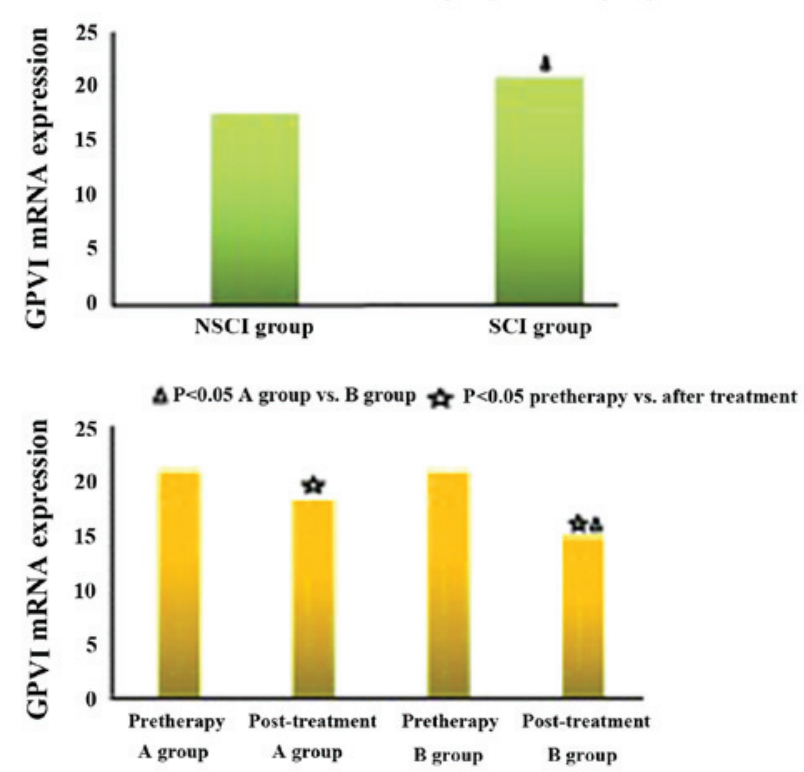

Figure 4. Comparison of GPVI mRNA expression between groups in diabetic nephropathy. GPVI, glycoprotein VI.

SCI patients were lower than those of NSCI patients, but the difference had no statistical significance. It indicates that it is rare for SCI patients to develop language dysfunction at an early stage. The neurologic dysfunction of patients in group B was significantly lower than those of patients in group A, while MoCA scores and clinical effectiveness were higher than group A. In MoCA scale, the combined treatment with alogliptin and motor imagery in hyperbaric oxygen was excellent regarding the function of visuospatial/executive, attention and concentration, which has much better improvement on neurocognitive function.

Previous findings have show that the platelet adhesion, aggregation and release of diabetic patients are enhanced, thus the risk of thrombosis is increased (29). Moreover, the platelets significantly change before and after the onset of cerebral infarction. Thus, the abnormality of platelet function may induce cerebral infarction. The changes in platelet count and platelet volume may be important factors for inducing cerebral infarction. The more severe the conditions and the larger the infarction area, the more obvious the change in platelet parameters $(30,31)$. In our preliminary study, in comparison with the NSCI group, the platelet count of SCI group decreased while the platelet distribution width increased (32). Poor blood glucose control may lead to the increase of HbAlc, histanoxia, the glycation of plasma, lowdensity lipoprotein-cholesterol, fibrinogen and platelets, the increase of blood glucose viscosity and free radical generation and the acceleration of atherosclerosis, which may induce cerebral infarction (33).
We also studied the evaluation of platelet function in patients by TEG and the secretion of platelet-associated cytokines. The mechanism of TEG occurs throughout the whole coagulation process, from coagulation to fibrinolysis. It is mainly applied to detect blood coagulation disorder and can comprehensively reflect the physical features of blood clot (including the rate of blood clot formation as well as the strength and stability of blood clot). Thus, it is an effective method to test coagulation changes of patients, and is characterized by small blood use, simple operation, short testing time and permanent preservation. Thus, it can be used to guide treatment and judge curative effect in clinic. In our study, SCI patients experienced a hypercoagulable state, and the combined treatment with alogliptin improved this hypercoagulable state.

GPVI is a platelet collagen receptor, that plays a key role in mediating signal transduction in platelets after collagen adhesion, activating GPIa-IIa and GPIIb-IIIa, promoting activity expression as well as accelerating platelet adhesiveness and thrombosis. GPVI levels of patients with acute coronary syndrome were higher than those of patients with stable angina pectoris $(34,35)$. The monitoring of GPVI levels in platelets may predict the risk for thrombosis $(36,37)$. 11-DH-TXB 2 is mainly from thromboxane A generated by platelets and is not formed in vitro. The content of $11-\mathrm{DH}-\mathrm{TXB}_{2}$ is stable in urine. Urine 11-DH-TXB ${ }_{2}$ is the best index for reflecting the release level of TXA2 in vivo, which may indirectly reflect the levels of platelet aggregation and activation (38). We found that the combined treatment of alogliptin and motor 
imagery in hyperbaric oxygen chamber can better promote thrombolysis. The GPVI expression and 11-DH-TXB ${ }_{2}$ level of combined treatment group B were significantly lower than those of group A.

In conclusion, the condition assessment and management of SCI patients greatly affects prognosis. SCI patients should have early prevention and early diagnosis. In particaular, diabetic patients with smoking, hyperlipidemia, hypertension, atrial fibrillation and carotid artery stenosis should regularly be followed-up irrespective of whether the desease is complicated with SCI. The treatment of DPP-IV inhibitor alogliptin combined with motor imagery in hyperbaric oxygen can better promote thrombolysis absorption, restore brain damage and improve neurocognitive dysfunction in DN patients with SCI. However, our study also has disadvantages. Due to small sample size, the curative effect on SCI patients at different infraction sites is not compared. The aforementioned disadvantages will be deeply discussed in subsequent studies in order to provide more scientific theoretical basis for the prevention and treatment of silent cerebral infraction in clinic.

\section{Acknowledgements}

We thank Mrs. Weixue Tang for technical assistance. She is work in the departments of Pathophysiology, Basic Medical College, Chongqing Medical University.

\section{References}

1. Dedov II and Shestakova MV: Cerebral vascular lesions in diabetes mellitus: Solved and unresolved questions. Zh Nevrol Psikhiatr Im S S Korsakova 115: 79-82, 2015 (In Russian).

2. Yi C, Liu W and Zhang Y: Correlation between metabolic syndrome and silent cerebral infarction: A cross-sectional study. J Third Mil Med Univ 33: 408-410, 2011.

3. Hashimoto M, Takashima Y, Uchino A, Yuzuriha T and Yao H: Dual task walking reveals cognitive dysfunction in community-dwelling elderly subjects: The Sefuri brain MRI study. J Stroke Cerebrovasc Dis 23: 1770-1775, 2014.

4. Jacquin A, Binquet C, Rouaud O, Graule-Petot A, Daubail B, Osseby GV, Bonithon-Kopp C, Giroud M and Béjot Y: Post-stroke cognitive impairment: High prevalence and determining factors in a cohort of mild stroke. J Alzheimers Dis 40: 1029-1038, 2014

5. Ma M, Hasegawa Y, Koibuchi N, Toyama K, Uekawa K, Nakagawa T, Lin B and Kim-Mitsuyama S: DPP-4 inhibition with linagliptin ameliorates cognitive impairment and brain atrophy induced by transient cerebral ischemia in type 2 diabetic mice. Cardiovasc Diabetol 14: 54, 2015.

6. Chan SY, Ou SM, Chen YT and Shih CJ: Effects of DPP-4 inhibitors on cardiovascular outcomes in patients with type 2 diabetes and end-stage renal disease. Int J Cardiol 218: 170-175, 2016.

7. Chen $\mathrm{CH}$, Chen SY, Wang V, Chen CC, Wang $\mathrm{KC}$, Chen $\mathrm{CH}$, Liu YC, Lu KC, Yip PK, Ma WY, et al: Effects of repetitive hyperbaric oxygen treatment in patients with acute cerebral infarction: A pilot study. ScientificWorldJournal 2012: 694703, 2012. doi: $10.1100 / 2012 / 694703$.

8. Yan J, Guo X, Jin Z, Sun J, Shen L and Tong S: Cognitive alterations in motor imagery process after left hemispheric ischemic stroke. PLoS One 7: e42922, 2012.

9. Zhu YC, Dufouil C, Tzourio C and Chabriat H: Silent brain infarcts: A review of MRI diagnostic criteria. Stroke 42: $1140-1145,2011$

10. Auriel E, Westover MB, Bianchi MT, Reijmer Y, MartinezRamirez S, Ni J, Van Etten E, Frosch MP, Fotiadis P, Schwab K, et al: Estimating total cerebral microinfarct burden from diffusion-weighted imaging. Stroke 46: 2129-2135, 2015.

11. Page SJ: Mental practice: A promising restorative technique in stroke rehabilitation. Top Stroke Rehabil 8: 54-63, 2001.

12. Petit LS, Pegna AJ, Mayer E and Hauert CA: Representation of anatomical constraints in motor imagery: Mental rotation of a body segment. Brain Cogn 51: 95-101, 2003.
13. Bushnell CD, Johnston DC and Goldstein LB: Retrospective assessment of initial stroke severity: Comparison of the NIH Stroke Scale and the Canadian Neurological Scale. Stroke 32: 656-660, 2001

14. Schmidt WP, Roesler A, Kretzschmar K, Ladwig KH, Junker R and Berger K: Functional and cognitive consequences of silent stroke discovered using brain magnetic resonance imaging in an elderly population. J Am Geriatr Soc 52: 1045-1050, 2004.

15. Yatsu FM and Shaltoni HM: Implications of silent strokes. Curr Atheroscler Rep 6: 307-313, 2004.

16. Nasreddine ZS, Phillips NA, Bédirian V, Charbonneau S, Whitehead V, Collin I, Cummings JL and Chertkow H: The Montreal Cognitive Assessment, MoCA: A brief screening tool for mild cognitive impairment. J Am Geriatr Soc 53: 695-699, 2005.

17. Akay OM, Ustuner Z, Canturk Z, Mutlu FS and Gulbas Z: Laboratory investigation of hypercoagulability in cancer patients using rotation thrombelastography. Med Oncol 26: 358-364, 2009.

18. Ohnishi $\mathrm{J}$ and Kudo Y: Clinical aspects of silent cerebral infarction - MRI findings and its distribution. Rinsho Shinkeigaku 31: 610-615, 1991 (In Japanese).

19. Shintani S, Shiigai T and Arinami T: Silent lacunar infarction on magnetic resonance imaging (MRI): Risk factors. J Neurol Sci 160: 82-86, 1998.

20. Mabuchi T, Kitagawa K, Kuwabara K, Takasawa K, Ohtsuki T, Xia Z, Storm D, Yanagihara T, Hori $M$ and Matsumoto M: Phosphorylation of cAMP response element-binding protein in hippocampal neurons as a protective response after exposure to glutamate in vitro and ischemia in vivo. J Neurosci 21 : 9204-9213, 2001.

21. Gault VA, Lennox R and Flatt PR: Sitagliptin, a dipeptidyl peptidase-4 inhibitor, improves recognition memory, oxidative stress and hippocampal neurogenesis and upregulates key genes involved in cognitive decline. Diabetes Obes Metab 17: 403-413, 2015.

22. James PB: Hyperbaric oxygenation in fluid microembolism. Neurol Res 29: 156-161, 2007.

23. Nazario $J$ and Kuffler DP: Hyperbaric oxygen therapy and promoting neurological recovery following nerve trauma. Undersea Hyperb Med 38: 345-366, 2011.

24. Gaggioli A, Meneghini A, Morganti F, Alcaniz M and Riva G: A strategy for computer-assisted mental practice in stroke rehabilitation. Neurorehabil Neural Repair 20: 503-507, 2006.

25. Walbruch B and Kalliainen L: The optimization of peripheral nerve recovery using cortical reorganization techniques: A retrospective study of wrist level nerve repairs. J Hand Ther 28: 341-345, 2015.

26. Pendlebury ST, Cuthbertson FC, Welch SJ, Mehta Z and Rothwell PM: Underestimation of cognitive impairment by Mini-Mental State Examination versus the Montreal Cognitive Assessment in patients with transient ischemic attack and stroke: A population-based study. Stroke 41: 1290-1293, 2010.

27. Tsai JC, Chen CW, Chu H, Yang HL, Chung MH, Liao YM and Chou KR: Comparing the sensitivity, specificity, and predictive values of the Montreal Cognitive Assessment and Mini-Mental State Examination when screening people for mild cognitive impairment and dementia in Chinese population. Arch Psychiatr Nurs 30: 486-491, 2016.

28. Lees RA, Hendry Ba K, Broomfield N, Stott D, Larner AJ and Quinn TJ: Cognitive assessment in stroke: Feasibility and test properties using differing approaches to scoring of incomplete items. Int J Geriatr Psychiatry: Aug 16, 2016 (Epub ahead of print).

29. Yamasaki Y, Kim YS and Kawamori R: Rationale and protocol of a trial for prevention of diabetic atherosclerosis by using antiplatelet drugs: Study of Diabetic Atherosclerosis Prevention by Cilostazol (DAPC study). Cardiovasc Diabetol 5: 16, 2006.

30. Lee JH, Kwon KY, Yoon SY, Kim HS and Lim CS: Characteristics of platelet indices, neutrophil-to-lymphocyte ratio and erythrocyte sedimentation rate compared with $\mathrm{C}$ reactive protein in patients with cerebral infarction: A retrospective analysis of comparing haematological parameters and $\mathrm{C}$ reactive protein. BMJ Open 4: e006275, 2014.

31. Du J, Wang Q, He B, Liu P, Chen JY, Quan H and Ma X: Association of mean platelet volume and platelet count with the development and prognosis of ischemic and hemorrhagic stroke. Int J Lab Hematol 38: 233-239, 2016.

32. Chen D, Huang X, Lu S, Deng H, Gan H, Du X and Weixue T: $\mathrm{RBP} 4 / \mathrm{Lp}-\mathrm{PLA} \mathrm{A}_{2} /$ Netrin-1 signaling regulation of cognitive dysfunction in diabetic nephropathy complicated with silent cerebral infarction. Exp Clin Endocrinol Diabetes: Jul 13, 2017 (Epub ahead of print). 
33. Dahl A, Lund C and Russell D: Atherosclerosis and cerebral infarction. Tidsskr Nor Laegeforen 127: 892-896, 2007.

34. Samaha FF, Hibbard C, Sacks J, Chen H, Varello MA, George T and Kahn ML: Density of platelet collagen receptors glycoprotein VI and alpha2betal and prior myocardial infarction in human subjects, a pilot study. Med Sci Monit 11: CR224-CR229, 2011

35. Kassaian SE, Fathi Y, Lotfi-Tokaldany M, Salarifar M, Alidoosti M, Haji-Zeinali AM, Aghajani H, Amirzadegan A, Nozari Y, Mortazavi SH, et al: Comparison of 1-year major adverse cardiac events in patients undergoing primary percutaneous coronary intervention receiving intracoronary bolus only versus intracoronary bolus plus infusion of glycoprotein IIb/IIIa inhibitors. Crit Pathw Cardiol 15: 89-94, 2016.

36. Motovska Z, Kvasnicka J, Widimsky P, Petr R, Hajkova J, Bobcikova P, Osmancik P, Odvodyova D and Katina S: Platelet glycoprotein GP VI $13254 \mathrm{C}$ allele is an independent risk factor of premature myocardial infarction. Thromb Res 125: e61-e64, 2010.
37. Ali-Hasan-Al-Saegh S, Mirhosseini SJ, Shahidzadeh A, Rahimizadeh E, Sarrafan-Chaharsoughi Z, Ghodratipour Z, Lotfaliani M, Rezaeisadrabadi M, Dehghan HR, Bireta C, et al: Appropriate bolus administration of glycoprotein IIb/IIIa inhibitors for patients with acute coronary syndromes undergoing percutaneous coronary intervention: Intracoronary or intravenous? A comprehensive and updated meta-analysis and systematic review. Kardiol Pol 74: 104-118, 2016.

38. Dharmasaroja PA and Sae-Lim S: Comparison of aspirin response measured by urinary 11-dehydrothromboxane B2 and VerifyNow aspirin assay in patients with ischemic stroke. J Stroke Cerebrovasc Dis 23: 953-957, 2014. 